\title{
¿CÓMO FORMAMOS PARA EL DESARROLLO DE CAPACIDADES CIUDADANAS Y PRODUCTIVAS EN EL PLAN NACIONAL DE FORMACIÓN DOCENTE DESDE LAS CIENCIAS SOCIALES?
}

Carlos Rodríguez Rivas Coordinador del CENICSH carlos.rodriguez@mined.gob.sv

Recepción: 4 de abril de 2016 Aceptación: 18 de abril de 2016 


\section{RESUMEN}

El presente artículo reúne las principales preocupaciones e ideas surgidas de las discusiones y del diálogo entre especialistas y expertos de ciencias sociales del Plan Nacional de Formación de Docentes en Servicio. Este proceso ha estado marcado por la construcción de un planteamiento pedagógico, metodológico y curricular desde el enfoque del desarrollo de capacidades ciudadanas y productivas. Las capacidades son potencialidades de la persona, que se constituyen a partir de la articulación de competencias, conocimientos, habilidades y modos de ser, con las cuales puede encararse situaciones complejas que presenta la vida cotidiana en sus diferentes dimensiones. Este enfoque implica la superación de un currículo fragmentado y centrado exclusivamente en la transmisión de conocimientos disciplinares. Este replanteamiento de la tarea de las ciencias sociales implica responder al porqué, para qué y cómo nos proponemos enseñar y aprender estas disciplinas.

\section{Palabras clave}

Capacidades ciudadanas - Capacidades productivas - Enseñanza de las ciencias sociales - Reflexión pedagógica - Formación docente

\section{ABSTRACT}

This paper summarizes the main concerns and ideas emerged from the discussions and dialogue between specialists and experts of social sciences of the National Plan of Teacher Training Service. This process has been marked by the construction of a pedagogical, methodological approach and curriculum development from the perspective of citizens and productive capacities. Capacities are potentialities of the person, which are formed from the articulation of skills, knowledge, skills and ways of being, which can be faced with complex situations presented in daily life in all its dimensions. This approach involves overcoming a fragmented curriculum and focused exclusively on the transmission of disciplinary knowledge. This rethinking of the task of the social sciences involves answering the why, what and how we intend to teach and learn these disciplines.

\section{Keywords}

Citizenship skills- productive capacities - Teaching of social sciences - Pedagogical reflection - Teacher training 


\title{
¿CÓMO FORMAMOS PARA EL DESARROLLO DE CAPACIDADES CIUDADANAS Y PRODUCTIVAS EN EL PLAN NACIONAL DE FORMACIÓN DOCENTE ${ }^{1}$ DESDE LAS CIENCIAS SOCIALES?
}

\author{
Carlos Rodríguez Rivas \\ Coordinador del CENICSH${ }^{2}$ \\ carlos.rodriguez@mined.gob.sv
}

\section{Introducción}

El desarrollo de capacidades ciudadanas y productivas constituye el horizonte desde el cual se impulsa la transformación del sistema educativo en sus diferentes dimensiones, niveles y modalidades educativas, así lo declara el Plan Nacional de Educación en Función de la Nación ${ }^{3}$. Esto expresa la convicción de que es necesario superar el modelo centrado en la transmisión de contenidos, dispersos, desarticulados y fragmentados, y las consecuencias de este modelo que condiciona el actual sistema educativo y que se expresan en el modelo de evaluación de los aprendizajes, la formación inicial docente, los programas de estudios, entre otros. En la nueva perspectiva trazada, la

\footnotetext{
${ }^{1}$ Plan Nacional de Formación de Docentes en Servicio en el Sector Público 2015-2019.

${ }^{2}$ Centro Nacional de Investigaciones en Ciencias Sociales y Humanidades delViceministerio de Ciencia y Tecnología del Ministerio de Educación de El Salvador.

${ }^{3}$ Ministerio de Educación. Ejes estratégicos del Plan Nacional de Educación en Función de la Nación. Educar para el desarrollo de capacidades productivas y ciudadanas. San Salvador: MINED, 2015.
} 
enseñanza de diferentes disciplinas o áreas curriculares tiene como finalidad última alcanzar un objetivo educativo superior, no la mera transmisión de contenidos disciplinares, la cual da unidad a los diferentes componentes y responde a las necesidades de desarrollo humano y productivo del país. Calidad educativa significa, entonces, alcanzar de forma satisfactoria el desarrollo pleno de este objetivo ulterior; con lo cual es necesario que la planta docente, la infraestructura escolar, el currículo nacional, los instrumentos de evaluación educativa, la escuela, el locus amplio de la educación, y toda la institucionalidad del sistema se oriente hacia el desarrollo de capacidades ciudadanas y productivas históricamente legítimas.

Desde luego que la educación es una instancia que posibilita el desarrollo humano, que brinda condiciones para la configuración de proyectos de vida diversos; no obstante, aunque la educación sea fuente de diversas posibilidades, en última instancia debe procurar que todas las personas se habiliten como buenos ciudadanos y que tengan las herramientas básicas que les permitan insertarse en el ámbito productivo, lo cual innegablemente, está a la vez de cualquier posterior elección o proyecto de vida que la persona construya. Estas tareas son irrenunciables para todo sistema educativo público o privado que se plantee con seriedad el desarrollo humano.

En este marco, el Ministerio de Educación impulsa el Plan Nacional de Formación de Docentes en Servicio en el Sector Público, el cual se ha planteado como la plataforma dinamizadora de la educación nacional, en tanto que consiste en un proceso de cualificación de la planta docente y es el punto de partida de la transformación curricular y de la reforma de la formación inicial de maestros. La estrategia del Plan de Formación se basa en la constitución de núcleos de expertos disciplinares de las diferentes áreas contempladas en el currículo nacional, para construir e impulsar la formación de especialistas docentes de alto nivel académico que posteriormente asuman la formación de todo el magisterio y una serie de tareas vinculadas a la evaluación y producción de materiales educativos. Los núcleos de expertos disciplinares se integran con los académicos más destacados del país, quienes 
en el desarrollo de la formación paulatinamente van fortaleciendo sus competencias pedagógicas y conociendo a profundidad la realidad y necesidades del sistema educativo, en el marco de la resolución de problemas asociados al proceso y en un diálogo e interlocución con los especialistas y docentes en formación. El Plan de Formación plantea una hoja de ruta para la formación docente y para el fortalecimiento de capacidades de los formadores de formadores.

En este sentido, es una estrategia de formación y de investigación de la educación nacional para la dinamización del currículo nacional en las ruta del desarrollo de capacidades ciudadanas y productivas, no solo en lo referido a los programas de estudio de los diferentes niveles y modalidades, sino al desarrollo pedagógico y metodológico desde el prisma disciplinar correspondiente a cada núcleo. En el área de ciencias sociales el Plan propone profundizar el dominio teórico y metodológico de los especialistas en formación desde cinco disciplinas científicas: Historia, Economía, Política, Antropología y Sociología, ${ }^{4}$ para lo cual se han diseñado dos especialidades: Ciencias Sociales para Primer y Segundo Ciclo de Educación Básica, y Ciencias Sociales para Tercer Ciclo y Educación Media. Al momento se han desarrollado 4 módulos de los 8 previstos para especialistas, tres para docentes de Estudios Sociales de Tercer ciclo y Media y uno para docentes de Primer y Segundo Ciclos de Educación Básica. El núcleo de expertos o planta de formadores de formadores está integrada por 30 académicos de las diferentes disciplinas, entre los cuales hay 12 doctores y 17 con nivel de maestría, cada uno con una amplia experiencia en la educación superior y una relevante producción científica.

El plan de estudios está compuesto por tres áreas, una dedicada a la problemática teórico y metodológica de las ciencias sociales en general, con la finalidad de sentar las bases de una concepción articulada de las ciencias

\footnotetext{
${ }^{4}$ Ministerio de Educación. Plan Nacional de Formación de Docentes en Servicio en el Sector Público. San Salvador: MINED, 2014.
} 
sociales; una segunda área donde se combinan conceptos básicos de las 5 disciplinas con el estudio de procesos desde el andamiaje conceptual previamente establecido y se problematiza las implicaciones metodológicas y de enseñanza asociadas y, finalmente, un área que prioriza el trabajo pedagógico y metodológico para la enseñanza de las ciencias sociales de cara al fortalecimiento de una sociedad más justa y democrática, lo cual no significa que en los módulos disciplinares no se problematice la enseñanza. Esta última área, por el contrario, está pensada para establecer un balance y una profundización de las discusiones planteadas a lo largo de la formación.

Apertura a la discusión teórica, reflexión metodológica desde el fortalecimiento disciplinar y flexibilidad para hacer ajustes y replanteamientos en el proceso son los principios que han guiado la actividad de los núcleos de expertos y especialistas. Así mismo, les ha guiado la firme convicción de que no hay fórmulas ni recetas absolutas, mucho menos didácticas generales que valgan si no se construye un sólido dominio disciplinar, y un planteamiento pedagógico claro que articule los diferentes campos con un profundo conocimiento de la realidad educativa nacional, la escuela y la comunidad.

La discusión permanente que el núcleo de expertos y especialistas de Ciencias Sociales ha tenido es cómo replantear la enseñanza de las referidas, con lo cual se está queriendo explicitar porqué, para qué y cómo nos proponemos enseñar estas disciplinas. Las primeras dos cuestiones tienen que ver con el planteamiento pedagógico, el cual esta esbozado en la propuesta de formación de capacidades ciudadanas y productivas, de donde el reto a este respecto consiste en profundizar este desarrollo y hacerlo viable en la escuela salvadoreña; con lo cual llegamos a la tercera cuestión, que consiste en descifrar y diseñar la metodología de enseñanza y evaluación adecuada al desarrollo de estas capacidades. Las modestas reflexiones que se presentan en seguida son el resultado del intercambio entre expertos, especialistas y docentes, quienes de forma comprometida han contribuido a perfilar esta primera síntesis y reflexión del proceso. 


\section{¿Qué son las capacidades ciudadanas y productivas?}

Las capacidades en un sentido general son potencialidades de la persona, que se constituyen a partir de la articulación de competencias (saber hacer concreto), conocimientos (diferentes disciplinas científicas, humanísticas y artísticas), habilidades (racionalidades y destrezas) y modos de ser (emociones, valores y carácter) con los cuales la puede encarar situaciones complejas que le presenta la vida cotidiana en sus diferentes dimensiones (interpersonales, familiares, comunitarias, económicas o políticas). Las capacidades ciudadanas pueden identificarse concretamente si consideramos tres aspectos básicos: las dimensiones de la ciudadanía, las esferas de acción ciudadana y el fundamento histórico de éstas.

En el primer punto nos referimos a aquellos componentes implicados en el concepto formal del buen ciudadano, es decir, aquel que es capaz de participar en el espacio público, en sus diferentes modalidades y escenarios, donde se dirimen conflictos y se discute el rumbo de las comunidades políticas o de la sociedad. Nos referimos a un concepto formal sin taras ideológicas, lo cual no implica rehuir a la integralidad de aspectos formales, que involucran no sólo el conocimiento del ordenamiento jurídico de la comunidad política, sino la capacidad de participar activamente en la gestión de conflictos (dimensión política); en este sentido, consideramos que cualquier plasmación concreta o definición holística de ciudadanía está integrada por la ciudadanía política, es decir, saber participar en la gestión de conflictos; la ciudanía legal que es conocer y asumir derechos y deberes; la ciudadanía social la cual consiste en asumir nuestro derechos sociales, culturales y económicos; la ciudadanía civil que es sentirse parte activa de la sociedad civil; ciudadanía para la diversidad que implica saber llevar y respetar las diferencias identitarias o interculturales; ciudadanía de la memoria que es saberse relacionar productivamente con el pasado; ciudadanía cosmopolita 
que significa asumirse como ciudadano del mundo ${ }^{5}$ en el marco de una mundialización de las relaciones sociales, ${ }^{6}$ y la ciudadanía económica y productiva donde tienen lugar las capacidades productivas que están definidas por una dimensión científico-técnica y otra ética.

El aspecto científico-técnico de las capacidades productivas se refiere a saber producir algo (lo cual implica conocimientos técnicos y científicos, y manejo de procedimientos formales los cuales se construyen desde los primeros niveles educativos y no están limitados a la educación superior); mientras que la dimensión ética es saber consumir bien, es decir, practicar un ética del consumo que tenga a la base el concepto de sustentabilidad, con lo cual se hacer referencia a consumir desde la responsabilidad de cuidar los recursos del entorno para mí y para las próximas generaciones. ${ }^{7}$

Por otra parte, una cuestión fundamental de este planteamiento consiste en la idea de que las capacidades productivas son capacidades ciudadanas, y esto es así porque una persona que no esté preparada para insertarse en el ámbito productivo, en esta doble perspectiva, es un ciudadano incompleto, a medias, que difícilmente podrá afirmar condiciones materiales para la realización de una ciudadanía plena. De esta concepción articulada de capacidades ciudadanas y productivas se sigue un principio curricular fundamental: que ambos pliegos posibilitantes (ciudadanos y productivos) no son asunto exclusivo de un conjunto particular de disciplinas; así las ciencias sociales no son las únicas responsables de la formación ciudadana, como las capacidades productivas no son únicamente la responsabilidad de las ciencias naturales y matemática; ${ }^{8}$ de ahí que en el marco de la dinamización curricular planteada desde el Plan de Formación sea fundamental plantearse

\footnotetext{
${ }^{5}$ Adela Cortina Orts. Ciudadanos del mundo: hacia una teoría de la ciudadanía. Madrid: Alianza, 2009.

${ }^{6}$ Antonio González Un solo mundo. Madrid: Bubok, 2008

${ }^{7}$ Jesús Conill Sancho. Horizontes de economía ética. Madrid: Tecnos, 2014; Riechmann, Jorge. Ética ecológica. Montevideo: Nordan-Comunidad, 2004; Hinkelamert, Franz. Hacia una economía para la vida. Cartago : Editorial Tecnológica de Costa Rica, 2008.

${ }^{8}$ Ministerio de Educación. Sistema Nacional de Evaluación. San Salvador: MINED, 2015.
} 
cómo las ciencias naturales y matemática aportan a la formación ciudadana igualmente cómo las ciencias sociales, las humanidades y las artes apoyan el desarrollo productivo de la persona.

Ahora bien, estas dimensiones de la ciudadanía se corresponden y se desarrollan en diferentes ámbitos de la sociedad que deben estar considerados para elaborar una propuesta pedagógica que las potencie desde el sistema educativo. Así, es importante pensar el desarrollo de estas capacidades desde la escuela, sin olvidar que este es solo uno de los posibles escenarios de ejecución de capacidades, por lo que hay que desarrollar estrategias que incorporen como ámbito de la ciudanía a la comunidad, la sociedad civil, el sistema electoral, el estado nación y las cosmopolis, es decir, el mundo. Una persona habilitada ciudadana y productivamente debe ser capaz de enfrentar situaciones complejas de forma compleja en cualquiera de estas esferas o ámbitos de la sociedad; lo cual significa que tenemos que diseñar estrategias educativas coherentes con esta ampliación de ámbitos de acción.

Sin embargo, estas capacidades asociadas a las dimensiones de la ciudadanía y a las esferas donde se despliegan, deben corresponderse con el momento histórico que la sociedad vive; y en este punto es donde estos aspectos formales que venimos planteando deben adquirir una concreción histórica, pues está claro que las necesidades de desarrollo ciudadano y productivo son diferentes según el momento histórico que la sociedad salvadoreña atraviesa; así, en la actualidad, debemos identificar un conjunto de capacidades a fortalecer que sean coherente con el escenario posterior a los Acuerdos Paz, a las dinámicas de la violencia de la sociedad actual, a la nueva dinámica de fortalecimiento de las relaciones mundiales, al escenario de la ciudadanía digital, entre otros aspectos que caracterizan la vida social.

Este planteamiento tiene una serie de consecuencias para el sistema educativo. Por ejemplo, si las capacidades productivas están asociadas a la formación científico-técnica, nuestro sistema educativo debe tener un carácter científico si quiere generar capacidades de este tipo y si quiere aportar a una sociedad productiva. La capacidad de producir y generar valor está condicio- 
nada por la manera en que nos relacionamos con las ciencias y con la manera en que solucionamos problemas básicos y de principios. Sin embargo, esto no se genera en cualquier condición, sino con una estructura educativa nacional fundamentada en dos pilares relacionados íntimamente, según sostiene el documento del Sistema Nacional de Evaluación: el sistema educativo público y el aparato productivo. Para lograr este asocio resulta esencial que dentro del sistema educativo exista un núcleo de desarrollo científico y tecnológico de cara a los problemas del aparato productivo, porque además el sistema educativo no puede definirse o generarse desde el sistema mismo, sino como un problema de nación asociado a la productividad del país y a la creación de medios de vida dignos para la sociedad en el marco de una discusión pública sobre sus prioridades y alcances. Esto quiere decir que los problemas de la creación de condiciones y medios de vida dignos no son solamente un problema económico y político sino educativo. ${ }^{9}$

Por otra parte, sostener que la formación de capacidades productivas solo puede ir junto con la habilitación de capacidades ciudadanas implica una concepción radicalmente diferente a la visión fragmentada y parcelada del conocimiento humano que se ha expresado en el currículo nacional; la distinción entre humanidades y ciencias sociales y ciencias naturales y matemática, no debe conducirnos a una separación de las dimensiones productivas y ciudadanas, más bien es en el sistema educativo, donde debemos aprender a construir capacidades que requieren de una íntima relación entre las disciplinas y los problemas concretos que plantea la realidad nacional. La habilitación de capacidades productivas y ciudadanas son dos aspectos de un solo asunto: la construcción de una ciudadanía crítica y responsable. A este replanteamiento integral debe sumarse el principio de que las capacidades no son meros contenidos de conocimiento, sino modos concretos de relacionarse socialmente, modos de colocarse ante problemáticas concretas; de ahí que la habilitación de capacidades no se resuelva con que el siste-

\footnotetext{
${ }^{9}$ Ministerio de Educación. Sistema Nacional de Evaluación. San Salvador: MINED, 2015.
} 
ma educativo transmita unos determinados conocimientos, lo relevante es que el sistema relacione esta enseñanza con la vida pública y la dimensión productiva.

Dicho esto podemos sostener que las capacidades ciudadanas y productivas son potencialidades de la persona referidas a la ciudadanía integral y a saber producir y consumir. Para el desarrollo de estas capacidades, tal y como sostuvimos anteriormente, es necesario formar una serie de competencias, racionalidades, conocimientos y un carácter que bien podrían resumirse en cuatro grandes campos: cognitivo, emocional, comunicacional e integrador. Algunos países como Colombia han avanzado a partir de este planteamiento y han formulado estándares básicos de competencias ciudadanas, ${ }^{10} \sin$ embargo, la experiencia salvadoreña nos dice que es necesario plantearse de entrada ir más allá de los estándares en dos sentidos; primero, porque no podemos contentarnos con una ciudadanía básica y, segundo, por es necesario historizar cada propuesta de formación ciudadana.

En el primer campo encontramos no solo conocimientos de diferente índole, sino lo más importante que son habilidades cognitivas como el pensamiento crítico, la meta cognición, la comprensión, la explicación y la capacidad reflexiva, entre otros. En el segundo orden nos referimos a la identificación de las diferencias, a la empatía, al manejo de las emociones, a la imaginación; en el ámbito comunicativo, a la capacidad de diálogo, a la elaboración argumentativa, al saber escuchar, y finalmente, nos referimos a competencias integradoras como el análisis del discurso, al talante ante situaciones de conflicto, es decir, modos que relacionan los primeros tres ámbitos y que al fin de cuentas materializan los modos complejos en que nos aproximamos o enfrentamos problemáticas.

Si pensamos este planteamiento a profundidad concluiremos fácilmente que dimensiones como la comunicacional no solo vienen dadas por el estudio del idioma o de la literatura, también la matemática es un lenguaje,

\footnotetext{
${ }^{10}$ Ministerio de Educación Nacional. Estándares básicos de competencias ciudadanas. Bogotá:
} Ministerio de Educación Nacional, 2003. 
y desde luego, las ciencias sociales aportan significativamente al desarrollo argumentativo. En el fondo, el desarrollo curricular hacia el enfoque de las capacidades $^{11}$ nos está obligando a superar la fragmentación del currículo nacional en áreas curriculares y competencias genéricas desarticuladas, así como a una enseñanza basada en la transmisión de contenidos. El enfoque pedagógico de las capacidades supone construir puentes entre los diferentes saberes y, desde luego, entre los docentes de diferentes asignaturas, así como desarrollar metodologías y estrategias de evaluación que permitan verificar los avances de la juventud en cuanto habilitación de capacidades. La llamada escuela tradicional tiene muy poco que aportar en este sentido, de allí que la estrategia de escuela inclusiva de tiempo pleno constituya un oportunidad invaluable para desarrollar estas capacidades que implícitamente significan ir más allá de la escuela y del aula.

Desde las Ciencias Sociales y las Humanidades, esto se traduce en varias preguntas: por ejemplo, ¿cómo la historia y otras disciplinas forman pensamiento crítico?, ¿pueden las ciencias sociales construir un carácter asociado al respeto a la diversidad?, ¿cuál es la tarea de las ciencias sociales de cara al desarrollo de capacidades productivas?, ¿cómo las humanidades aportan al desarrollo de una ética del consumo?, entre otras. Es importante decir, en la dinámica social actual, y en la transformación de la experiencia del tiempo, que la tarea de la educación no es tanto garantizar unos conocimientos sólidos sino unas capacidades para enfrentar el cambio. ${ }^{12}$

\footnotetext{
${ }^{11}$ Martha Nussbaum. Crear capacidades: propuesta para el desarrollo humano. Barcelona: Paidós Ibérica, 2012; Gough, Ian. "El enfoque de las capacidades de M. Nussbaum: un enfoque comparado con nuestra teoría de las necesidades humanas" Papeles de Relaciones Eco sociales y Cambio Global. No 100 (2007): 177-202.

${ }^{12}$ Zygmunt Bauman . Los retos de la educación en la modernidad liquida. Barcelona: Gedisa, 2007.
} 


\section{Diálogo y reflexión con el magisterio: hacía un diagnóstico de lo que ocurre en la escuela}

Un primer objetivo trazado para el desarrollo del enfoque de las capacidades en el marco del Plan de Formación ha sido introducir la temática de la formación ciudadana en el proceso, para reflexionar con los maestros y maestras sobre qué ocurre al respecto en la escuela. A partir del desarrollo del módulo 3 Sociedad, política y poder para especialistas de Tercer Ciclo y Media fue más clara la necesidad de orientar la formación en esta dirección, por lo que se presentó a los docentes un recorrido sobre los diferentes modelos de formación ciudadana que se han impulsado a partir de firmada la paz, ${ }^{13}$ esto como la antesala de un diagnóstico realizado por ellos donde la preguntas generadoras fueron: ¿qué hacemos desde la escuela para formar ciudadanía? y ¿cómo están nuestros jóvenes en materia de formación ciudadana?

Como resultado de esta reflexión se identificaron, en primer lugar, aquellas estrategias que se utilizan en el aula para formar ciudadanía, con lo cual se constató que en el ideario docente son preponderantes dos dimensiones de la ciudadanía, la legal y la política. En el primer caso, la estrategia consiste en informar al joven de sus derechos, y en el segundo caso, a la emulación de acciones del sistema electoral, como elecciones estudiantiles o la estrategia de diputados por un día, que muestran una concepción restringida de la ciudadanía política, limitada al espacio electoral. Las acciones o bien reafirman metodologías tradicionales centradas en el maestro y en la transmisión o bien son acciones desvinculadas de las asignaturas o programas de estudio. A la base de estas acciones no hay una reflexión pedagógica sobre la formación ciudadana.

En la información surgida de los grupos focales pudo identificarse que en el sistema educativo sobreviven diferentes políticas de formación

\footnotetext{
${ }^{13}$ Rodríguez Rivas, Carlos. Formación ciudadana: elementos para la discusión desde las ciencias sociales. Presentación desarrollada en el marco del módulo dos de formación de expertos, 5 de diciembre de 2015.
} 
ciudadana producto de los tres grandes planteamientos educativos construidos desde la finalización de la guerra civil, Reforma educativa en marcha, ${ }^{14}$ Plan $2021^{15}$ y Plan Social Educativo Vamos a la Escuela. ${ }^{16}$ En la escuela salvadoreña confluyen diferentes apuestas como la formación en valores, la educación para la paz, la educación para la vida y la educación moral y cívica, lo que nos hace concluir que es necesario el desarrollo de una política nacional de formación ciudadana que una vez por todas unifique los esfuerzos dispersos y que aclare los fundamentos pedagógicos, y que, desde luego, defina cómo se traduce esto en términos de formación docente. En el marco de la dispersión de acciones, conviene decir que el enfoque de las capacidades tiene que enfrentarse al prejuicio dominante que privilegia la transmisión de conocimientos, el entendimiento de qué es un valor determinado, lo cual no implica necesariamente su práctica, mucho menos la simulación de escenarios complejos donde puedan aplicarse los conocimientos o los valores.

Asimismo, es igualmente relevante tematizar desde la ampliación de la noción de ciudadanía propuesta por el enfoque de las capacidades todos los reduccionismos vigentes en el sistema, por ejemplo, el planteado por la formación cívica, es decir, aquella que se preocupa por el resguardo de una identidad y de unos valores nacionales, pero no de las capacidades necesarias para hacer política de forma propositiva e innovadora en los diferentes ámbitos de participación ciudadana y mucho menos de la coexistencia de identidades diversas. Un planteamiento homogenizador como el que está a la base de la formación moral y cívica tradicional es atentatorio de una perspectiva de convivencia y respeto a la diversidad. ${ }^{17}$

\footnotetext{
${ }^{14}$ Ministerio de Educación. Lineamientos del Plan Decenal 1995-2005. San Salvador: MINED, 1999.

15 Ministerio de Educación. Plan Nacional de Educación 2021: Metas y políticas para construir el país que queremos. San Salvador: MINED, 2005.

${ }^{16}$ Ministerio de Educación. Plan Social Educativo Vamos a la escuela. San Salvador: MINED, 2009.

${ }^{17}$ Guillermo Hoyos. "Ética, interculturalidad y pluralismo” en Toro Bernardo. Educación, valores y ciudadanía. Madrid: OEI, 2010.
} 
Por otra parte, el discurso dominante de la formación de valores favorece una perspectiva subjetiva, interiorista, sustentada en la tradición, es decir, una noción comunitarista de ciudadanía, lo que importa es la buena persona, ser bueno como en el pasado, por lo que la meta es recuperar los valores que se pierden y no tanto construir personas que participan activamente en los problemas de la sociedad y en la construcción de nuevos horizontes de valoración. El culto al pasado que está a la base de este planteamiento ignora el proceso ininterrumpido de transformación y de cambio de los horizontes de valoración, lo cual de suyo no es algo negativo, sino la dinámica de autoreproducción de la sociedad. Más bien habría que plantearse trabaja con los jóvenes cómo vamos cambiando los valores y los vamos replanteando según los nuevos escenarios de convivencia.

El diagnóstico también arroja valiosos aportes sobre el estado de la juventud en materia de formación ciudadana, los valores dominantes en la juventud, el desprestigio de la profesión docente, los actores que inciden y el problema de la violencia, como aspecto liminar de las acciones pedagógicas. Es muy importante puntualizar, asimismo, que los docentes se asumen como agentes estratégicos en la trasformación de estas realidades, no obstante, carecer de la formación, las herramientas y el espacio pedagógico para emprender esta ardua tarea. Es tarea irrenunciable del sistema educativo, tomar medidas urgentes a este respecto.

Una pregunta que irremediablemente hubo que plantear fue: ¿qué es ciudadanía?, o ¿qué entendemos por ciudadanía? Porque es evidente que es necesario tener claridad de esto para emprender acciones pedagógicas coherentes. En este punto el enfoque de fortalecimiento disciplinar del Plan de Formación fue muy útil, ya que encarar esta cuestión requiere de conceptos básicos de la ciencia política, de filosofia política y de las ciencias sociales en general, así como nociones mínimas sobre el decurso de la discusión al respecto. Ello nos permitió concluir que un maestro de estudios sociales que tenga por tarea formar ciudadanía debe tener claridad meridiana de qué tipo de ciudadano se desea formar y de las implicaciones teóricas como antesala 
para discutir las estrategias metodológicas y las acciones pedagógicas pertinentes. El currículo vigente establece como competencia genérica de los estudios sociales formar una ciudadanía crítica y responsable, pero no dice en ninguna parte en qué consiste eso. Discutir con los maestros este tipo de cuestiones aclara y da elementos para su práctica pedagógica.

Tematizada la problemática de la formación ciudadana, planteado qué tipo de ciudadano nos estamos planteando formar y realizado el breve diagnóstico, decidimos aproximarnos al planteamiento curricular vigente. Los resultados de este ejercicio no fueron menos enriquecedores. En primer lugar constatamos cómo el tema de la formación ciudadana esta vagamente planteado en la Ley general de educación y cómo los Fundamentos curriculares de la educación naciona $1^{18}$ no plantean ningún desarrollo. El problema es que, muy a pesar del replanteamiento por competencias del currículo nacional, la formación ciudadana y en valores planteada desde la Reforma educativa en marcha nunca pasó de ser una actividad marginal. Esto se expresa en los programas de estudio sociales y cívica, donde los contenido están planteados sin asocio a la formación de competencias, y las metodologías procuran garantizar un proceso dinámico de trasmisión de conocimientos o, en el mejor de los casos, procesos de construcción de los conocimientos centrados en el estudiante, pero no de cara a la formación de competencias, de habilidades o destrezas. Todo el currículo nacional sigue centrado en los contenidos de conocimiento, así sea que los transmita el docente o los construya el estudiante.

Por otra parte, como ya se dijo, las denominadas competencias genéricas del currículo nacional expresan una visión totalmente fragmentada de la educación, al grado que las competencias comunicativas son tarea exclusiva del área de lenguaje y literatura, así como las modestas competencias de participación activa y responsable son dominio de las ciencias sociales.

${ }^{18}$ Ministerio de Educación. Fundamentos curriculares de la educación nacional. San Salvador: MINED, 1994. 
El enfoque de las capacidades desarrollado líneas atrás supone superar esta fragmentación de las áreas curriculares, así como descentrar la acción pedagógica de los contenidos del conocimiento, y finalmente, concretar y justificar históricamente las capacidades ciudadanas y productivas a potenciar, superando las formulaciones ultra genéricas del currículo nacional.

Esto último expresa tres tareas fundamentales para la dinamización del currículo nacional en esta perspectiva, y da sustento a la estrategia del Plan de Formación Docente, a saber, en el fortalecimiento disciplinar, un manejo más fino de los conceptos básicos de las ciencias sociales de cara a los problemas pedagógicos, lo cual pone en agenda de la reflexión docente no solo la tarea de reflexionar sobre su práctica en el aula, sino el andamiaje curricular sobre el cual está montada esta práctica. Finalmente, desde esta problematización, ensayar desarrollos didácticos y metodológicos que procuren tender puentes con el trabajo que se realiza desde otras áreas curriculares. De esta manera, el proceso de formación en servicio se convierte en una estrategia que no solo impacta en las capacidades docentes, sino en una fuente privilegiada de ensayo y validación de nuevas prácticas pedagógicas asociadas al desarrollo del enfoque de las capacidades.

\section{El fortalecimiento disciplinar y la problematización de las ciencias sociales de cara al enfoque de las capacidades}

El diálogo y la interlocución entre docentes especialistas y expertos formadores llevan consigo la necesidad de replantear constantemente la propuesta de formación en materia de ciencias sociales. En este sentido uno de los primeros resultados ha sido reelaborar el plan de formación para la especialidad. En un primer momento, los planes de formación estaban centrados en los conocimientos, sin duda, el enfoque de asociar andamiajes conceptuales con el estudio de casos o procesos concretos era adecuado pero insuficiente, ya 
que era necesario conectar esto con las problemáticas educativas que tienen los maestros.

De este modo concluimos que cada módulo debía tener tres componentes, conceptos básicos disciplinares, estudio de casos a partir de los conceptos disciplinares, y problemática educativa y pedagógica desde los aspectos anteriores y desde el enfoque de las capacidades. La idea fue evitar una idea secuencial, muy por el contrario la dificultad ha sido establecer una relación entre estos tres componentes a lo largo del proceso. Así se reelaboró cada plan atendiendo a los siguientes aspectos:

- Primero, hay que partir o construir un entendimiento de qué son las ciencias sociales y cómo sus problemas metodológicos y epistemológicos están vinculados a la enseñanza de estas (Teoría social y análisis de la sociedad). Por ejemplo, es muy productivo discutir desde los modelos de crítica que ha desarrollado la teoría social, sobre qué queremos decir cuando hablamos de formar pensamiento crítico desde la escuela.

- Segundo, fortalecer los conocimientos en economía para saber cómo funciona la producción y cómo podemos desarrollar una ética del consumo (Sociedad, economía y desarrollo).

- Tercero, fortalecer los conocimientos básicos sobre las comunidades y estructuras políticas, revisar desde este prisma la historia política de El Salvador, problematizar los diferentes modelos de ciudadanía surgidos desde cada estructura política y ver cuáles son las deudas en materia ciudadana y qué se puede hacer desde la escuela (Sociedad, política y poder).

- Cuarto, hay que ver qué es la cultura, las identidades y la dimensión sociocultural para superar las perspectivas: biologicista, culturalista y sociologista sobre la identidad, que fundamentan la invisibilización, promueven la discriminación y exclusión de grupos identitarios, y fortalecer la capacidad ciudadana de la tolerancia y el respeto a la diversidad. 
- Quinto, desarrollar la capacidad de reflexionar críticamente sobre el pasado desde el presente, a través de una revisión de procesos históricos que configuraron El Salvador en que vivimos.

- Sexto, entender la sociedad donde vivimos y cómo está organizada para reflexionar sobre los mecanismos y estructuras que generan desigualdades, y reflexionar sobre cómo la escuela pueden encarar esta problemática o evitar constituirse en otro mecanismo más de producción de desigualdades.

- Séptimo, qué es la democracia, cuáles son los diferentes modelos, pensar la democracia salvadoreña, sus grandes retos, y tematizar las tarea de formar capacidades ciudadanas y productivas en una sociedad democrática, profundizando en las herramientas que podemos utilizar y ajustarlas al enfoque de las capacidades.

- Octavo, integrar todos estos elementos para diseñar una respuesta metodológica a la enseñanza de las ciencias sociales que dé respuesta al reto del desarrollo de capacidades ciudadanas y productivas, estableciendo desde un enfoque holístico mecanismos de integración disciplinar y de elaboración metodológica profunda, más allá de las técnicas formales y de las didácticas generales.

La intención de este replanteamiento es aclarar cómo cada una de las cinco ciencias sociales (historia, política, economía, antropología y sociología) involucradas en el fortalecimiento disciplinar aportan individual y colectivamente a la formación de diferentes capacidades ciudadanas y productivas. Los resultados de este ejercicio aún son modestos pero relevantes. Hemos constatado cómo algunos objetivos de las acciones pedagógicas que se desarrollan son sumamente abstractos, por ejemplo, qué significa formar pensamiento crítico, o qué es una educación inclusiva, y desde estas dos primeras preguntas: ¿Son las metodologías que usamos relevantes para formar pensamiento crítico, o se quedan cortas, son abstractas? ¿Podemos mejorar las estrategias metodológicas utilizadas para generar un espacio inclusivo? La discusión teó- 
rica asociada a los problemas de la escuela es sumamente rica para generar un proceso real de especialización docente, el cual no consiste simplemente en que conozcan las ciencias sociales, más allá de los modestos estudios sociales, sino en que tengamos la capacidad de revisar, ajustar y diseñar estrategias metodológicas inspiradas en los andamiajes teóricos que revisamos y que respondan al reto de formar capacidades ciudadanas y productivas.

El desarrollo de competencias para conocer e interpretar hechos y fenómenos sociales, para realizar análisis críticos, para formular hipótesis y sustentaciones que den cuenta de una construcción propia o intersubjetiva de significados, ${ }^{19}$ para aproximarse responsablemente a la comprensión y a la acción o toma de decisiones respecto de una situación problemática concreta son objetivos que están a la base de acciones pedagógicas que vamos proponiendo a los docentes. Ha sido dificil superar la formación centrada en la exposición magistral, pero poco a poco se van diseñando y validando mecanismos que permite que los docentes en formación o bien pongan en práctica estas competencias o bien se den cuenta, a partir de un conflicto cognitivo, de la relevancia que tiene el desarrollo de estas para la enseñanza de las ciencias sociales.

Ha sido muy valioso para el desarrollo de esta formación asumir que construimos esquemas y estructuras de conocimiento a partir de esquemas preexistentes, por lo que no puede ignorarse la comparación crítica de lo nuevo con lo viejo, ponernos en una situación de conocimiento significa no solo adquirir nuevas informaciones sino compararlas críticamente con las anteriores; el diálogo en torno a los pre saberes es central siempre y cuando no sea un momento protocolar, sino un proceso finamente diseñado para generar conflictos cognitivos, sin olvidar que ha sido fuente importante para saber cuál es el nivel de conocimiento y formación del magisterio, así como un veta importante para trazarse acciones que respondan a los vacíos e inconsistencias de procesos de formación anteriores.

\footnotetext{
${ }^{19}$ Carolina Valencia. "Pedagogía de las ciencias sociales" Revista de Estudios Sociales. № 19 (2004): 9-95.
} 


\section{El desarrollo metodológico desde el fortalecimiento disciplinar}

Desarrollar una cierta reflexión crítica para examinar a fondo nuestra práctica pedagógica ${ }^{20}$ para identificar su alcance, sus límites y enfocarla pedagógicamente de acuerdo a un propósito ha sido el punto de partida de la propuesta de desarrollo metodológico que venimos ensayando. Desarrollo metodológico desde el fortalecimiento disciplinar no es conocer primero la disciplina y después reflexionar sobre cómo enseñarla. El reto ha consistido en establecer relaciones permanentes entre estos dos aspectos.

Ahora bien, en este proceso ha sido muy útil partir de diagnósticos de lo que hacemos y cómo están los jóvenes, los estudiantes. Así se procedió cuando abordamos el módulo Sociedad, política y poder y así se diseñó el de Historia de El Salvador en el siglo XX para especialistas de tercer ciclo y media. En este último fue importante partir de algunas conclusiones sobre lo que ocurre en la escuela de cara a la enseñanza de la historia:

- Agobio docente por la ingente cantidad de temas y el poco tiempo para el abordaje.

- Desconocimiento de investigaciones recientes y la falta de manejo de fuentes primarias.

- Aburrimiento y poco interés que genera en los estudiantes esta temática.

- Uso de las tradicionales técnicas del dictado o el copiado que vuelve monótona y vacía la enseñanza de la historia.

- Falta de uso de herramientas y fuentes no textuales que enriquecen y den un rostro vivo a la historia.

- Ausencia de relación entre la enseñanza de la historia y el desarrollo de competencias cognitivas, emocionales y comunicativas.

${ }^{20}$ Ibidem. 
- Concentración en la historia política y económica expresada en los programas y libros de texto.

- Desvinculación de los procesos históricos locales, nacionales y globales.

A partir de esto, se diseñó una propuesta pedagógica desde el enfoque de las capacidades para la enseñanza de la historia que dé sustento al proceso de reflexión metodológica que pretendemos desarrollar con los especialistas; esto es lo que denominamos desarrollo del enfoque de las capacidades desde las disciplinas. Se parte de la convicción de que la historia es fundamental para la formación de ciudadanía, para lo cual es necesario superar aquella práctica pedagógica en la que el profesor es el autor y el presentador de un texto con un saber cerrado, presentado como verdadero, mientras que el protagonismo de los alumnos se reduce a ser meros receptores de unas informaciones que muchas veces no saben por qué deben aprenderlas, memorizarlas o repetirlas. ${ }^{21}$ Los puntos más importantes de este desarrollo pedagógico se resumen a continuación.

Crítica de la idea reduccionista del tiempo histórico: con esto se pretende superar la idea de la historia como una sucesión lineal de acontecimientos que poco o nada tiene que ver con el presente. Preguntarse cuáles son los efectos de la historia sobre el presente, se convierte en una tarea central y además permite hacer una selección de temáticas relevantes para la enseñanza de esta disciplina y nos conduce a una concepción de la historia como ciencia del presente. Ello con la intención de convertir el trabajo histórico y la enseñanza de esta disciplina en un recurso para construir vínculos en la sociedad, comprender los problemas del presente, promover la tolerancia y la convivencia, así como una perspectiva de largo aliento, muy útil para el desarrollo de un carácter mesurado, de una ciudadanía responsable.

Pensamiento crítico y formación ciudadana: preguntarse por un planteamiento pedagógico para la enseñanza de la historia implica preguntarse

${ }^{21}$ Joan Pagés. Ciudadanía y enseñanza de la historia. Reseñas de enseñanza de la historia. $\mathrm{N}^{\circ} 1$ (Octubre 2003): 11-42. 
por su sentido educativo. La historia se aborda desde su importancia para la formación de ciudadanía cuyas dimensiones implica el fortalecimiento de capacidades y competencias a tres órdenes: lo cognitivo, lo emotivo y lo comunicativo. En el primer orden resalta en otras el aporte de la historia a la formación de pensamiento crítico; en el segundo, es importante mostrar que la historia nos afecta y en este sentido nos compromete de algún modo con el presente (empatía), y finalmente, la historia como un discurso que fortalece la dimensión discursiva y argumentativa, central en los procesos de diálogo e interlocución.

Fortalecimiento de las capacidades de investigación: en este punto, se considera que el método histórico es una herramienta que puede fortalecer los procesos de investigación sobre la práctica pedagógica. Asimismo, el método histórico puede aportar al fortalecimiento del diseño de metodologías y estrategias didácticas, así como al desarrollo del pensamiento crítico en los jóvenes desde la práctica del método histórico. La idea no es enseñar métodos de investigación como un fin en sí, sino para fortalecer el desarrollo de metodologías de la enseñanza.

Enfoque situación-problema:22 esta estrategia es coherente con la crítica a la idea reduccionista del tiempo histórico. Consiste en plantear en forma de problema un tema, en este caso un tema de la historia, para que, a partir de varios elementos puedan visualizarse propuestas de solución o de abordaje de una determinada situación problemática para el presente, con la intención de generar aprendizajes significativos. Se parte del esbozo de una problemática actual, por ejemplo, la violencia, se caracteriza su forma actual, para tener un punto de partida desde el presente, para la posterior indagación en el pasado de sus diversas formas de manifestación. Esto con la intención de lograr una situación de empatía que parta de lo cotidiano

\footnotetext{
${ }^{22}$ Verónica Mora Villafuerte y Fernando Flores Castillo. "La enseñanza de la historia a través de la situación-problema. Una apropiación metodológica para el docente vista desde su práctica en el aula." X Congreso Nacional de Investigación Educativa (Veracruz, 21 al 25 de septiembre de 2009).
} 
a la elaboración de referentes históricos que posibilitan la construcción de esquemas conceptuales que permitan reflexionar sobre el presente. La idea es partir de conocimientos previos, para ser problematizado con la revisión de nuevas fuentes, nuevas interpretaciones y enfoques, para construir un ambiente de discusión con los docentes o los estudiantes. Las dinámicas fundamentales son de interlocución, pero a la luz de la rigurosa revisión de fuentes primarias o secundarias.

Lo visto y lo no visto en la historia: el uso de fuentes no textuales: en concordancia con el punto anterior se revisan las prácticas al uso. La fuente fundamental del historiador ha sido el documento, el texto; sin documentos oficiales, no se podía escribir historia. Sin embargo, este paradigma metodológico es insostenible en la actualidad. El uso de alternativas como las fuentes orales, de imágenes, el cine y la música, los monumentos o lugares de memoria, no sólo nos plantean construir escenarios más complejos, sino volver más atractiva la enseñanza de la historia, así como construir procesos de autoidentificación de los docentes o los estudiantes con el pasado.

Deconstrucción de las historias nacionales y abordaje crítico de la historia oficial: se trata de partir del cuestionamiento de una historia nacional u oficial que pretende ser globalizadora o totalizante, y peor la versión definitiva de los acontecimientos. En primer lugar, la historia también trata de cómo nos ven otros, y cómo nuestra historia es historia también del mundo. Este es un punto importante donde pueden conectarse con la historia discusiones de otras disciplinas, como la política; por ejemplo, la discusión sobre el debilitamiento de los estados-nación y cómo esto impacta en el enfoque de la resolución de problemas que estamos planteando. Por otra parte, cuando se habla de historia oficial usualmente se hace referencia a aquel discurso o narrativa histórica que busca establecer pautas para la justificación histórica del presente, de un estado de cosas o en algunos casos del Estado; pero la historia también debe verse como un esfuerzo por comprender críticamente el pasado. Esto nos lleva a concluir varias cuestiones; primero, que la historia debe contribuir a la formación de un ciudadano del mundo, que entiende 
que los problemas locales y nacionales tienen relación con los globales; y, en segundo lugar, que la enseñanza de la historia debe convertirse en un proceso de investigación para el docente y para los jóvenes.

Partiendo de los puntos señalados como ejes de la propuesta pedagógica, los cuales se presentan como aspectos a discutir con los docentes planteamos que es posible darle sustento a la reflexión metodológica que para nosotros tiene tres componentes: cómo está planteado programáticamente la enseñan del saber (programas de estudio), cuáles y cómo son las herramientas que utilizamos (didáctica) y cómo son nuestras practica pedagógicas y el espacio en el que las desarrollamos.

En el primer punto es importante invitar a los docentes a reflexionar sobre los límites de los programas, por ejemplo, siempre en cuanto a la enseñanza de la historia es fácil constatar que estos privilegian la historia política y económica en detrimento de otras dimensiones de la vida social que igual pueden historiarse o que constituyen variables fundamentales para explicar lo político o económico. En el segundo momento, consideramos importante problematizar las herramientas didácticas al uso, como las línea del tiempo y o las cronologías, las cuales a partir del planteamiento pedagógico pueden complejizarse, por ejemplo, incorporando un idea del tiempo histórico o del decurso histórico signada por las rupturas más que por las continuidades; asimismo el uso de fuentes de otra índole representa un reto importante para los maestros en esta dirección. En esta perspectiva reflexión metodológica significa ajustar la caja de herramientas desde la disciplinas y desde el planteamiento pedagógico.

Finalmente, es importante decir que el desarrollo metodológico desde el fortalecimiento disciplinar implica un ethos docente signado por la constante preocupación por el aprendizaje efectivo del otro. De ahí que esto no tiene sentido si no logramos que nuestros maestros asuman esta tarea como una tarea permanente y como parte de su actividad cotidiana, de su ethos. Esta ética se ve fortalecida si ellos cuentan con las herramientas de investigación de las ciencias sociales ya que el desarrollo didáctico no puede 
seguirse reduciendo a unas capacitaciones que se dan cada cierto tiempo, el docente tiene tareas diarias que demandan de una didáctica entendida no como un conjunto de técnicas o herramientas ya establecidas, sino como una forma de conocimiento que el docente debe cultivar a través de la investigación. ${ }^{23}$

\section{Principales desafíos estratégicos y hoja de ruta del proceso}

Esta reflexión quedaría inconclusa si no se plantearan algunos retos y desafíos fundamentales de cara a convertir el Plan de Formación Docente en una auténtica plataforma de dinamización de la educación nacional. Consideramos que dentro de esas tareas a corto y mediano plazo se encuentran el establecimiento de ámbitos de articulación entre la enseñanza de las ciencias sociales y otras disciplinas o áreas curriculares; desarrollo y ensayo de estrategias e instrumentos de evaluación asociados; desarrollo de metodologías adecuadas al nivel y modalidad educativa; la integración de las tecnologías educativas al planteamiento pedagógico; la elaboración de materiales educativos, y la integración con la Escuela Inclusiva de Tiempo Pleno. La tarea de las ciencias sociales en relación a esto es muy importante, no solo en lo que respecta a la enseñanza de estas disciplinas, sino a la construcción y establecimiento de un diálogo con las otras disciplinas.

\footnotetext{
${ }^{23}$ Joan Pagés. "Aprender a enseñar historia y ciencias sociales: el currículo y la didáctica de las ciencias sociales”. Pensamiento educativo. Vol. 30 (Julio 2002): 225-269.
} 


\section{REFERENCIAS BIBLIOGRÁFICAS}

\section{Documentos oficiales}

Ministerio de Educación. Ejes estratégicos del Plan Nacional de Educación en Función de la Nación. Educar para el desarrollo de capacidades productivas y ciudadanas. San Salvador: MINED, 2015

Plan Nacional de Formación de Docentes en Servicio en el Sector Público. San Salvador: MINED, 2014

. Sistema Nacional de Evaluación. San Salvador: MI-

NED, 2015

Ministerio de Educación. Lineamientos del Plan Decenal 1995-2005. San Salvador: MINED, 1999

. Plan Nacional de Educación 2021: Metas y políticas para construir el país que queremos. San Salvador: MINED, 2005

Plan Social Educativo Vamos a la escuela. San Salvador: MINED, 2009

. Fundamentos curriculares de la educación nacional. San

Salvador: MINED, 1994

\section{Libros y artículos}

Bauman, Zygmunt. Los retos de la educación en la modernidad liquida. Barcelona: Gedisa, 2007

Conill Sancho, Jesús. Horizontes de economía ética. Madrid:Tecnos, 2014

Cortina Orts, Adela. Ciudadanos del mundo: hacia una teoría de la ciudadanía. Madrid: Alianza, 2009

Gough, Ian. "El enfoque de las capacidades de M. Nussbaum: un enfoque comparado con nuestra teoría de las necesidades humanas" Papeles de Relaciones Eco sociales y Cambio Global. No 100 (2007): 177-202 
González, Antonio. Un solo mundo. Madrid: Bubok, 2008

Hinkelamert, Franz. Hacia una economía para la vida. Cartago: Editorial Tecnológica de Costa Rica, 2008.

Hoyos, Guillermo. "Ética, interculturalidad y pluralismo" en Toro Bernardo. Educación, valores y ciudadanía. Madrid: OEI, 2010

Nussbaum, Martha. Crear capacidades: propuesta para el desarrollo humano. Barcelona: Paidós Ibérica, 2012

Ministerio de Educación Nacional. Estándares básicos de competencias ciudadanas. Bogotá: Ministerio de Educación Nacional, 2003

Mora Villafuerte, Verónica y Flores Castillo, Fernando. "La enseñanza de la historia a través de la situación-problema. Una apropiación metodológica para el docente vista desde su práctica en el aula." $X$ Congreso Nacional de Investigación Educativa (Veracruz, 21 al 25 de septiembre de 2009)

Pagés, Joan. Ciudadanía y enseñanza de la historia. Reseñas de enseñanza de la historia. No 1 (Octubre 2003): 11-42

"Aprender a enseñar historia y ciencias sociales: el currículo y la didáctica de las ciencias sociales". Pensamiento educativo. Vol. 30 (Julio 2002): 225-269

Riechmann, Jorge. Ética ecológica. Montevideo: Nordan-Comunidad, 2004

Rodríguez Rivas, Carlos. Formación ciudadana: elementos para la discusión desde las ciencias sociales. Presentación desarrollada en el marco del módulo dos de formación de expertos, 5 de diciembre de 2015, inédito.

Valencia, Carolina. "Pedagogía de las ciencias sociales" Revista de Estudios Sociales. No 19 (2004): 9-95 